TRANSACTIONS OF THE

AMERICAN MATHEMATICAL SOCIETY

Volume 363, Number 8, August 2011, Pages 4463-4474

S 0002-9947(2011)05393-6

Article electronically published on March 23, 2011

\title{
THE GRONE-MERRIS CONJECTURE
}

\author{
HUA BAI
}

\begin{abstract}
In spectral graph theory, the Grone-Merris Conjecture asserts that the spectrum of the Laplacian matrix of a finite graph is majorized by the conjugate degree sequence of this graph. We give a complete proof for this conjecture.
\end{abstract}

The Laplacian of a simple graph $G$ with $n$ vertices is a positive semi-definite $n \times n$ matrix $L(G)$ that mimics the geometric Laplacian of a Riemannian manifold; see $\$ 1$ for definitions, and [2, 14] for comprehensive bibliographies on the graph Laplacian. The spectrum sequence $\lambda(G)$ of $L(G)$ can be listed in non-increasing order as

$$
\lambda_{1}(G) \geq \lambda_{2}(G) \geq \cdots \geq \lambda_{n-1}(G) \geq \lambda_{n}(G)=0 .
$$

For two non-increasing real sequences $\mathbf{x}$ and $\mathbf{y}$ of length $n$, we say that $\mathbf{x}$ is majorized by $\mathbf{y}$ (denoted $\mathbf{x} \preccurlyeq \mathbf{y}$ ) if

$$
\sum_{i=1}^{k} x_{i} \leq \sum_{i=1}^{k} y_{i} \text { for all } k \leq n \text {, and } \sum_{i=1}^{n} x_{i}=\sum_{i=1}^{n} y_{i} .
$$

This notion was introduced because of the following fundamental theorem.

Theorem 1 (Schur-Horn Dominance Theorem [18, 11). There exists a Hermitian matrix $H$ with diagonal entry sequence $\mathbf{x}$ and spectrum sequence $\mathbf{y}$ if and only if $\mathbf{x} \preccurlyeq \mathbf{y}$.

In particular, if $\mathbf{d}(G)=\left(d_{1}, d_{2}, \ldots, d_{n}\right)^{T}$ is the non-increasing degree sequence of $G$, which coincides with the diagonal entry sequence of the Laplacian matrix $L(G)$, the Schur-Horn Dominance Theorem implies that $\mathbf{d}(G) \preccurlyeq \lambda(G)$. Grone [7] improves this majorization result: if $G$ has at least one edge, then $\left(d_{1}+1, d_{2}, \ldots, d_{n-1}, d_{n}-\right.$ $1)^{T} \preccurlyeq \lambda(G)$.

For a non-negative integral sequence $\mathbf{d}$, we define its conjugate degree sequence as the sequence $\mathbf{d}^{\prime}=\left(d_{1}^{\prime}, d_{2}^{\prime}, \ldots, d_{n}^{\prime}\right)^{T}$ where

$$
d_{k}^{\prime}:=\#\left\{i: d_{i} \geq k\right\} .
$$

Another important majorization relation is the following.

Theorem 2 (Gale-Ryser [6, 17]). There exists a $(0,1)$-matrix $A$ with row and column sum vectors $\mathbf{r}$ and $\mathbf{c}$ if and only if $\mathbf{r} \preccurlyeq \mathbf{c}^{\prime}$.

Received by the editors November 12, 2009 and, in revised form, December 11, 2009, January 1, 2010 and May 28, 2010.

2010 Mathematics Subject Classification. Primary 15A42; Secondary 05C50.

Key words and phrases. Grone-Merris Conjecture, Laplacian matrix, majorization, split graph, Courant-Fischer-Weyl Min-Max Principle, simplicial complex.

The author was partially supported by NSF grant DMS-0604866.

(C)2011 American Mathematical Society 
Applying this to the adjacency matrix of $G$ immediately gives that $\mathbf{d}(G) \preccurlyeq \mathbf{d}^{\prime}(G)$. In 1994, Grone and Merris [8, 9] raised the natural question whether the Laplacian spectrum sequence and the conjugate degree sequence are majorization comparable.

Grone-Merris Conjecture. For any graph $G$, the Laplacian spectrum is majorized by the conjugate degree sequence

$$
\lambda(G) \preccurlyeq \mathbf{d}^{\prime}(G) .
$$

In this paper, we give a complete proof to the Grone-Merris Conjecture. As a consequence, we have the double majorization $\mathbf{d}(G) \preccurlyeq \lambda(G) \preccurlyeq \mathbf{d}^{\prime}(G)$.

See 3 for a partial result in this direction, as well as [19, 12, 13, 1] for proofs in the special cases. Also see [3] for a generalization to simplicial complexes, which is still open.

\section{The LAPLACIAN MATRIX AND THE MAJORIZATION RELATION}

Let $G=(V, E)$ be a simple finite graph with $n=|V|$ vertices. We write $i \sim j$ when the $i$-th vertex is adjacent to the $j$-th vertex, and we let $d_{i}$ denote the degree of the $i$-th vertex.

The Laplacian matrix $L(G)$ of the graph $G$ is the $n \times n$ matrix defined by

$$
L(G)_{i j}= \begin{cases}d_{i} & \text { if } i=j \\ -1 & \text { if } i \sim j \\ 0 & \text { otherwise }\end{cases}
$$

We can also express the Laplacian as $L(G)=D-A$, where $D$ is the diagonal matrix defined by the degree sequence, and $A$ is the adjacency $(0,1)$-matrix of the graph.

It is well known that $L(G)$ is positive semi-definite, since it corresponds to the quadratic form

$$
\mathbf{x}^{T} L(G) \mathbf{x}=\sum_{i \sim j}\left(x_{i}-x_{j}\right)^{2} \text { for } \mathbf{x}=\left(x_{1}, \ldots, x_{n}\right)^{T} \in \mathbb{R}^{n} .
$$

Let $\lambda=\left(\lambda_{1}, \lambda_{2}, \ldots, \lambda_{n}\right)^{T}$ be the non-increasing spectrum sequence of the Laplacian matrix $L(G)$. The smallest eigenvalue is $\lambda_{n}=0$, with eigenvector $\mathbf{1}_{n}=$ $(1,1, \ldots, 1)^{T}$.

Given two vectors $\mathbf{x}=\left(x_{1}, \ldots, x_{n}\right)^{T}$ and $\mathbf{y}=\left(y_{1}, \ldots, y_{n}\right)^{T}$ in $\mathbb{R}^{n}$, rearrange their components in non-increasing order as

$$
x_{[1]} \geq x_{[2]} \geq \cdots \geq x_{[n]}, \quad y_{[1]} \geq y_{[2]} \geq \cdots \geq y_{[n]} .
$$

We say that $\mathbf{x}$ is majorized by $\mathbf{y}$, and write $\mathbf{x} \preccurlyeq \mathbf{y}$, if

$$
\sum_{i=1}^{k} x_{[i]} \leq \sum_{i=1}^{k} y_{[i]} \text { for all } 1 \leq k \leq n \text {, and } \sum_{i=1}^{n} x_{i}=\sum_{i=1}^{n} y_{i} .
$$

We will make use of the following majorization inequality.

Theorem 3 (Fan [4]). If $H_{1}$ and $H_{2}$ are Hermitian matrices, then

$$
\lambda\left(H_{1}+H_{2}\right) \preccurlyeq \lambda\left(H_{1}\right)+\lambda\left(H_{1}\right) .
$$




\section{SPLIT GRAPHS}

A graph is split (also called semi-bipartite in [12]) if its vertices can be partitioned into a clique $V_{1}$ and a co-clique $V_{2}$. This is equivalent to saying that the subgraph induced by $V_{1}$ is complete, and that the subgraph induced by $V_{2}$ is an independent set. See [5, 20, 15, 10] for many characterizations and properties of split graphs.

Given a split graph $G=(V, E)$, let $N=\left|V_{1}\right|$ be the size of the clique, and $M=\left|V_{2}\right|$ be the size of the co-clique. Let $\delta(G)$ be the maximum degree of vertices in $V_{2}$. Clearly $\delta(G) \leq N$, and the Laplacian matrix of the split graph $G$ is of the form

$$
L(G)=\left(\begin{array}{cc}
K_{N}+D_{1} & -A \\
-A^{T} & D_{2}
\end{array}\right)
$$

where $K_{N}$ is the Laplacian matrix of the complete graph on $N$ vertices, where $D_{1}$ and $D_{2}$ are diagonal matrices with diagonal entries the vertex degrees of $V_{1}, V_{2}$, respectively, and where $A$ is the adjacency matrix for edges between $V_{1}$ and $V_{2}$.

The Laplacian matrix is symmetric, and therefore Hermitian.

Theorem 4 (Courant-Fischer-Weyl [16]). Let the $n \times n$ matrix $H$ be Hermitian, with eigenvalues $\lambda_{1} \geq \lambda_{2} \geq \cdots \geq \lambda_{n}$. Then

$$
\lambda_{k}=\max _{\operatorname{dim}(S)=k} \min _{0 \neq x \in S} \frac{\langle H x, x\rangle}{\langle x, x\rangle}=\min _{\operatorname{dim}(S)=n-k+1} \max _{0 \neq x \in S} \frac{\langle H x, x\rangle}{\langle x, x\rangle},
$$

where the $\max ($ resp. $\min )$ is taken over all $k$-dimensional (resp. $(n-k+1)$ dimensional) subspaces of $\mathbb{R}^{n}$.

We first investigate the Laplacian spectrum of a split graph.

Proposition 5. If $G$ is a split graph of clique size $N$, then

$$
\lambda_{N-1}(G) \geq N \geq \delta(G) \geq \lambda_{N+1}(G) .
$$

Moreover, if $\lambda_{N}(G) \geq N$, then

$$
\sum_{i=1}^{N} d_{i}^{\prime}=N^{2}+\operatorname{Tr}\left(D_{1}\right) .
$$

Proof. To prove the inequalities involving $\lambda_{N-1}(G)$ and $\lambda_{N+1}(G)$ by the CourantFischer-Weyl Min-Max Principle, it suffices to find an $(N-1)$-dimensional (resp. $M$-dimensional) subspace for which the action of $L(G)$ has a desirable lower (resp. upper) bound. There are natural candidates.

Let $P \subset \mathbb{R}^{M+N}$ be the $(N-1)$-dimensional subspace consisting of all vectors of the form $\left(\begin{array}{c}u \\ \mathbf{0}_{M}\end{array}\right)$ with $u \in \mathbb{R}^{N}$ and $u \perp \mathbf{1}_{N}$. Then for any unit vector $u \in \mathbb{R}^{n}$,

$$
\left\langle L(G)\left(\begin{array}{c}
u \\
\mathbf{0}_{M}
\end{array}\right),\left(\begin{array}{c}
u \\
\mathbf{0}_{M}
\end{array}\right)\right\rangle=\left\langle\left(K_{N}+D_{1}\right) u, u\right\rangle=N+\left\langle D_{1} u, u\right\rangle \geq N .
$$

Similarly, consider the $M$-dimensional subspace $Q \subset \mathbb{R}^{M+N}$ consisting of all vectors of the form $\left(\begin{array}{c}\mathbf{0}_{N} \\ u\end{array}\right)$ with $u \in \mathbb{R}^{M}$. Then for any unit vector $u$,

$$
\left\langle L(G)\left(\begin{array}{c}
\mathbf{0}_{N} \\
u
\end{array}\right),\left(\begin{array}{c}
\mathbf{0}_{N} \\
u
\end{array}\right)\right\rangle=\left\langle D_{2} u, u\right\rangle \leq \delta(G) .
$$

This proves the part of our first statement that $\lambda_{N-1}(G) \geq N \geq \delta(G) \geq \lambda_{N+1}(G)$. 
When $\lambda_{N}(G) \geq N$, we assert that the degree of any vertex in the clique $V_{1}$ is at least $N$. For this, suppose that our assertion is false, namely that there exists a vertex $v_{0} \in V_{1}$ with degree less than $N$. Then this vertex $v_{0}$ is adjacent to none of the vertices of the co-clique $V_{2}$. Consequently $G$ can be regarded as a split graph with new clique $V_{1} \backslash\left\{v_{0}\right\}$ and new co-clique $V_{2} \cup\left\{v_{0}\right\}$. The size of the new clique is $\widetilde{N}=N-1$. Applying the first part of the proposition, we obtain that

$$
\lambda_{N}(G)=\lambda_{\widetilde{N}+1}(G) \leq \widetilde{N}=N-1,
$$

which is a contradiction.

For a conjugating pair of non-negative integral sequences, the partial sum of one sequence can be computed in a different way as

$$
\sum_{i=1}^{N} d_{i}^{\prime}=\sum_{i=1}^{N} \sum_{j=1}^{M+N} \chi\left(d_{j} \geq i\right)=\sum_{j=1}^{M+N} \min \left(d_{j}, N\right),
$$

where $\chi$ is the characteristic function. The second part of the proposition now follows from the observation that

$$
\sum_{j=1}^{M+N} \min \left(d_{j}, N\right)=\sum_{j \in V_{1}} N+\sum_{j \in V_{2}} d_{j}=N^{2}+\operatorname{Tr}\left(D_{2}\right)=N^{2}+\operatorname{Tr}\left(D_{1}\right) .
$$

The next lemma will play an essential role in our proof of the Grone-Merris Conjecture. Its proof is presented in the next section.

Lemma 6. Assume that $G$ is a split graph of clique size $N$. If either $\lambda_{N}(G)>N$ or $\lambda_{N}(G)=N>\delta(G)$, then the $N$-th inequality of the Grone-Merris Conjecture holds, namely

$$
\sum_{i=1}^{N} \lambda_{i} \leq \sum_{i=1}^{N} d_{i}^{\prime}
$$

\section{THE Hомотору METHOD}

This section is devoted to proving Lemma 6. We adopt a homotopy method, following an idea of Katz [12] in his proof of the Grone-Merris Conjecture for 1regular semi-bipartite graph.

Let $\alpha \in[0,1]$. Define an $(M+N) \times(M+N)$ matrix $L_{\alpha}$ as

$$
L_{\alpha}=(1-\alpha)\left(\begin{array}{cc}
K_{N}+M & -J_{N \times M} \\
-J_{M \times N} & N
\end{array}\right)+\alpha\left(\begin{array}{cc}
K_{N}+D_{1} & -A \\
-A^{T} & D_{2}
\end{array}\right),
$$

where $J_{M \times N}$ denotes the $M \times N$ matrix whose entries are all equal to 1 .

Note that $L_{1}=L(G)$ is the matrix we are interested in, and that $L_{0}$ is the Laplacian of a complete split graph. The spectrum of $L_{0}$ is well understood:

Lemma 7. The Laplacian spectrum of the complete split graph of clique size $N$ and co-clique size $M$ is

$$
\left\{(M+N)^{(N)}, N^{(M-1)}, 0^{(1)}\right\},
$$


where $P^{(Q)}$ denotes $Q$ copies of the number $P$. The eigenspace corresponding to the eigenvalue $N$ consists of all vectors of the form $\left(\begin{array}{c}\mathbf{0}_{N} \\ v\end{array}\right)$, where $v$ is $M$-dimensional and $v \perp \mathbf{1}_{M}$; the eigenspace corresponding to the eigenvalue $(M+N)$ is spanned by the orthogonal vectors

$$
\left(\mathbf{0}_{i-1}, M+N-i,-\mathbf{1}_{M+N-i}\right)^{T}, \quad 1 \leq i \leq N .
$$

Lemma 8. If $\lambda_{N}(G)>N$ or $\lambda_{N}(G)=N>\delta(G)$, then

$$
\lambda_{N+1}^{(\alpha)} \leq N<\lambda_{N}^{(\alpha)} \text { for all } 0 \leq \alpha<1 .
$$

Proof. We again make use of the Courant-Fischer-Weyl Min-Max Principle. Recall that the $M$-dimensional subspace $Q \subset \mathbb{R}^{M+N}$ consists of all vectors of the form $\left(\begin{array}{c}\mathbf{0}_{N} \\ u\end{array}\right)$ with $u \in \mathbb{R}^{M}$. Then for any unit vector $u$,

$$
\begin{aligned}
\left\langle L_{\alpha}\left(\begin{array}{c}
\mathbf{0}_{N} \\
u
\end{array}\right),\left(\begin{array}{c}
\mathbf{0}_{N} \\
u
\end{array}\right)\right\rangle & =(1-\alpha)\langle N u, u\rangle+\alpha\left\langle D_{1}(u), u\right\rangle \\
& \leq(1-\alpha) N+\alpha \delta(G) \leq N .
\end{aligned}
$$

Therefore, the $(N+1)$-th largest eigenvalue $\lambda_{N+1}^{(\alpha)}$ is at most $N$.

For the eigenvalue $\lambda_{N}^{(\alpha)}$, let $\tilde{P}$ be the $N$-dimensional subspace which is spanned by the eigenvectors of $L_{1}$ corresponding to the $N$ largest eigenvalues. Clearly $\tilde{P} \perp$ $\mathbf{1}_{M+N}$. For any unit vector $v \in \tilde{P}$, we know from Lemma 7 that $\left\langle L_{0}(v), v\right\rangle \geq N$. Moreover,

$$
\begin{aligned}
\left\langle L_{\alpha}(v), v\right\rangle & =\alpha\left\langle L_{1}(v), v\right\rangle+(1-\alpha)\left\langle L_{0}(v), v\right\rangle \\
& \geq \alpha \lambda_{N}(G)+(1-\alpha) N \geq N .
\end{aligned}
$$

Therefore, the $N$-th largest eigenvalue $\lambda_{N}^{(\alpha)}$ is at least $N$.

We next proceed to show that the inequality on $\lambda_{N}^{(\alpha)}$ is strict when $0 \leq \alpha<1$. We already know that $\lambda_{N}^{(0)}=M+N$. If $\lambda_{N}^{(\alpha)}=N$ for some $0<\alpha<1$, then the above arguments show that necessarily

$$
\lambda_{N}(G)=N,\left\langle L_{1} v, v\right\rangle=N, \text { and } L_{0}(v)=N v .
$$

The first condition $\lambda_{N}(G)=N$ implies that $\delta(G)<N$, from our assumption on $\lambda_{N}(G)$; the third condition $L_{0}(v)=N v$ implies that $v$ is a unit vector in $\operatorname{Ker}\left(L_{0}-N\right)$, thus in turn a unit vector of $Q$. Then

$$
\left\langle L_{1} v, v\right\rangle \leq \delta(G)<N
$$

which contradicts the second condition $\left\langle L_{1} v, v\right\rangle=N$.

We now consider all possible $N$-dimensional subspaces $\left(\begin{array}{c}I_{N} \\ V^{(\alpha)}\end{array}\right) \subseteq\left(\mathbf{1}_{M+N}\right)^{\perp}$, where $V^{(\alpha)}$ is an $M \times N$ matrix. Here the notation of the subspace means that the subspace is spanned by the column vectors of the matrix $\left(\begin{array}{c}I_{N} \\ V^{(\alpha)}\end{array}\right)$. 
Lemma 9. If the subspace $\left(\begin{array}{c}I_{N} \\ V^{(\alpha)}\end{array}\right) \subseteq\left(\mathbf{1}_{M+N}\right)^{\perp}$ is an invariant subspace of $L_{\alpha}$, then the matrix $V^{(\alpha)}$ solves the quadratic matrix equation

$$
\begin{aligned}
& V^{(\alpha)}\left[(1-\alpha) M+\alpha\left(N+D_{1}\right)\right] \\
= & -(1-\alpha) J_{M \times N}-\alpha A^{T}+\alpha\left[D_{2}-V^{(\alpha)}\left(J_{N \times M}-A\right)\right] V^{(\alpha)} .
\end{aligned}
$$

In terms of matrix entries, this means that

$$
v_{j i}^{(\alpha)}=\frac{-(1-\alpha)-\alpha \chi(i \sim j)+\alpha\left(f_{j} v_{j i}-\sum_{i^{\prime}=1}^{N} \sum_{j^{\prime} \nsim i^{\prime}} v_{j i^{\prime}}^{(\alpha)} v_{j^{\prime} i}^{(\alpha)}\right)}{(1-\alpha) M+\alpha\left(N+d_{i}\right)},
$$

where the non-negative integers $d_{i}, f_{j}$ are the entries of the diagonal matrices

$$
D_{1}=\operatorname{Diag}\left(d_{1}, d_{2}, \ldots, d_{N}\right), \quad D_{2}=\operatorname{Diag}\left(f_{1}, f_{2}, \ldots, f_{M}\right) .
$$

Proof. It is easy to see that the orthogonal complement in $\mathbb{R}^{M+N}$ of the subspace $\left(\begin{array}{c}I_{N} \\ V^{(\alpha)}\end{array}\right)$ is the subspace $\left(\begin{array}{c}-V^{(\alpha)^{T}} \\ I_{M}\end{array}\right)$. If the subspace $\left(\begin{array}{c}I_{N} \\ V^{(\alpha)}\end{array}\right)$ is an invariant subspace of $L_{\alpha}$, then so is its orthogonal complement, since $L_{\alpha}$ is a symmetric matrix.

The $L_{\alpha}$-invariance property is equivalent to the existence of two square matrices $X_{\alpha}$ and $Y_{\alpha}$ such that

$$
L_{\alpha}\left(\begin{array}{cc}
I_{N} & -V^{(\alpha)^{T}} \\
V^{(\alpha)} & I_{M}
\end{array}\right)=\left(\begin{array}{cc}
I_{N} & -V^{(\alpha)^{T}} \\
V^{(\alpha)} & I_{M}
\end{array}\right)\left(\begin{array}{cc}
X_{\alpha} & 0 \\
0 & Y_{\alpha}
\end{array}\right) .
$$

By comparison of the corresponding four block matrices, we immediately obtain that

$$
\begin{aligned}
X_{\alpha} & =K_{N}+(1-\alpha) M+\alpha D_{1}-\left[(1-\alpha) J_{N \times M}+\alpha A\right] V^{(\alpha)}, \\
Y_{\alpha} & =(1-\alpha) N+\alpha D_{2}+\left[(1-\alpha) J_{M \times N}+\alpha A^{T}\right] V^{(\alpha)^{T}},
\end{aligned}
$$

together with a quadratic matrix equation for $V^{(\alpha)}$ :

$$
\begin{aligned}
& V^{(\alpha)}\left[K_{N}+(1-\alpha) M+\alpha D_{1}\right]+(1-\alpha) J_{M \times N}+\alpha A^{T} \\
= & \left\{(1-\alpha) N+\alpha D_{2}+V^{(\alpha)}\left[(1-\alpha) J_{N \times M}+\alpha A\right]\right\} V^{(\alpha)} .
\end{aligned}
$$

Because $\left(\begin{array}{c}I_{N} \\ V^{(\alpha)}\end{array}\right) \perp \mathbf{1}_{M+N}$, the entries of $V^{(\alpha)}$ satisfy that

$$
\sum_{j=1}^{M} v_{j i}^{(\alpha)}=-1 \text { for any } 1 \leq i \leq N
$$

This condition, in terms of matrices, is equivalent to $J_{N \times M} V^{(\alpha)}=-J_{N \times N}$. This implies that $V^{(\alpha)} K_{N}=\left[N+V^{(\alpha)} J_{N \times M}\right] V^{(\alpha)}$, with which the above quadratic matrix equation can be simplified to

$$
\begin{aligned}
& V^{(\alpha)}\left[(1-\alpha) M+\alpha\left(N+D_{1}\right)\right] \\
= & -(1-\alpha) J_{M \times N}-\alpha A^{T}+\alpha\left[D_{2}-V^{(\alpha)}\left(J_{N \times M}-A\right)\right] V^{(\alpha)} .
\end{aligned}
$$


The quadratic matrix equation is complicated, and is almost impossible to be solved explicitly. Fortunately, we do not have to do so.

From Lemma 8 and the assumption on $\lambda_{N}(G)$, we know that

$$
\lambda_{N+1}^{(\alpha)} \supsetneqq \lambda_{N}^{(\alpha)} \text { for all } \alpha \in[0,1] .
$$

Thus the subspace spanned by the eigenvectors of $L_{\alpha}$ corresponding to the $N$ largest eigenvalues is unique. Assume that this subspace is given by $\left(\begin{array}{c}I_{N} \\ V^{(\alpha)}\end{array}\right)$, so that the matrix $V^{(\alpha)}$ is well defined.

Lemma 10. The map $V^{(\alpha)}:[0,1] \rightarrow \mathbb{R}^{M \times N}$ is a continuous function of $\alpha$, for the usual metric of $\mathbb{R}^{M \times N}$.

Proof. Assume that $\alpha_{n}$ is a sequence in [0,1] such that $\alpha_{n} \rightarrow \alpha$ as $n \rightarrow \infty$.

According to the algebraic multiplicity of eigenvalues of $L_{\alpha}$, there exist positive integers $l=l(\alpha)$ and $i_{1}, \ldots, i_{l}\left(i_{0}=0\right.$ by convention) such that $i_{1}+i_{2}+\cdots+i_{l}=N$ and

$$
\lambda_{i_{1}+\cdots+i_{k-1}+1}^{(\alpha)}=\cdots=\lambda_{i_{1}+\cdots+i_{k-1}+i_{k}}^{(\alpha)}>\lambda_{1+i_{1}+\cdots+i_{k-1}+i_{k}}^{(\alpha)}, \quad \forall 1 \leq k \leq l .
$$

Let $\left\{u_{i}^{\beta}\right\}_{i=1}^{M+N}$ be an orthonormal basis consisting of the eigenvectors corresponding to the eigenvalues $\lambda_{i}^{(\beta)}$ for any $\beta \in[0,1]$, and $\left\{Z_{k}^{\alpha_{n}}\right\}_{k=1}^{l},\left\{W_{k}^{\alpha}\right\}_{k=1}^{l}$ denote two sequences of monotonic subspaces of $\mathbb{R}^{M+N}$ given by

$$
Z_{k}^{\alpha_{n}}=\operatorname{span}\left\{u_{i}^{\alpha_{n}}: i \leq i_{1}+\cdots+i_{k}\right\}, W_{k}^{\alpha}=\operatorname{span}\left\{u_{i}^{\alpha}: i>i_{1}+\cdots+i_{k-1}\right\} .
$$

By the Courant-Fischer-Weyl Min-Max Principle,

$$
\min _{0 \neq u \in Z_{k}^{\alpha_{n}}} \frac{\left\langle L_{\alpha_{n}}(u), u\right\rangle}{\langle u, u\rangle}=\lambda_{i_{1}+\cdots+i_{k}}^{\left(\alpha_{n}\right)} \rightarrow \lambda_{i_{1}+\cdots+i_{k}}^{(\alpha)} \quad \text { as } n \rightarrow \infty
$$

and

$$
\max _{0 \neq v \in W_{k+1}^{\alpha}} \frac{\left\langle L_{\alpha}(v), v\right\rangle}{\langle v, v\rangle}=\lambda_{1+i_{1}+\cdots+i_{k}}^{(\alpha)} \lessgtr \lambda_{i_{1}+\cdots+i_{k}}^{(\alpha)} .
$$

It follows that $Z_{k}^{\alpha_{n}} \cap W_{k+1}^{\alpha}=\{0\}$ and $Z_{k}^{\alpha_{n}} \oplus W_{k+1}^{\alpha}=\mathbb{R}^{M+N}$ when $n$ is sufficiently large. Moreover, we obtain that $Z_{l}^{\alpha_{n}}=\bigoplus_{k=1}^{l}\left(Z_{k}^{\alpha_{n}} \cap W_{k}^{\alpha}\right)$ from

$$
\operatorname{dim}\left(Z_{k}^{\alpha_{n}} \cap W_{k}^{\alpha}\right)=\operatorname{dim}\left(Z_{k}^{\alpha_{n}}\right)+\operatorname{dim}\left(W_{k}^{\alpha}\right)-(M+N)=i_{k}
$$

Consider a basis of the subspace $Z_{k}^{\alpha_{n}} \cap W_{k}^{\alpha}$ which consists of unit vectors of the form

$$
u_{k, n, s}=\cos \left(\theta_{k, n, s}\right) u_{i_{1}+\cdots+i_{k-1}+s}^{\alpha}+\sin \left(\theta_{k, n, s}\right) w_{k, s}, \quad 1 \leq s \leq i_{k},
$$

for some unit vector $w_{k, s} \in W_{k+1}^{\alpha}$. Necessarily $\lim _{n \rightarrow \infty} \sin \left(\theta_{k, n, s}\right)=0$, since $\left\langle L_{\alpha_{n}}\left(u_{k, n, s}\right), u_{k, n, s}\right\rangle \geq \lambda_{i_{1}+\cdots+i_{k}}^{\left(\alpha_{n}\right)}$ and

$$
\begin{aligned}
\left\langle L_{\alpha}\left(u_{k, n, s}\right), u_{k, n, s}\right\rangle & =\cos ^{2}\left(\theta_{k, n, s}\right) \lambda_{i_{1}+\cdots+i_{k}}^{\alpha}+\sin ^{2}\left(\theta_{k, n, s}\right)\left\langle L_{\alpha}\left(w_{k, s}\right), w_{k, s}\right\rangle \\
& \leq \cos ^{2}\left(\theta_{k, n, s}\right) \lambda_{i_{1}+\cdots+i_{k}}^{(\alpha)}+\sin ^{2}\left(\theta_{k, n, s}\right) \lambda_{i_{1}+\cdots+i_{k}+1}^{(\alpha)}
\end{aligned}
$$

Any vector $u \in Z_{l}^{\alpha_{n}}$ can now be expressed as

$$
u=\sum_{k=1}^{l} \sum_{s=1}^{i_{k}} c_{k, s}\left[\cos \left(\theta_{k, n, s}\right) u_{i_{1}+\cdots+i_{k-1}+s}^{\alpha}+\sin \left(\theta_{k, n, s}\right) w_{k, s}\right] .
$$


Assume that the maximum of $\left|c_{k, s}\right|$ is achieved at $\left|c_{k_{0}, s_{0}}\right|$. Due to the orthogonality of $\left\{u_{i}^{\alpha}\right\}_{i}$, the absolute value of the coefficient of $u_{i_{1}+\cdots+i_{k_{0}-1}+s_{0}}^{\alpha}$ is at most $\|u\|$. But when $n$ is sufficiently large, it is at least

$$
\left|c_{k_{0}, s_{0}}\right| \cdot\left(\left|\cos \left(\theta_{k_{0}, n, s_{0}}\right)\right|-\sum_{k=1}^{l} \sum_{s=1}^{i_{k}}\left|\sin \left(\theta_{k, n, s}\right)\right|\right) \geq \frac{\left|c_{k_{0}, s_{0}}\right|}{2} .
$$

Hence $\left|c_{k_{0}, s_{0}}\right| \leq 2\|u\|$. For any given vector $v \in W_{l+1}^{\alpha}$, we see that

$$
|\langle u, v\rangle|=\left|\sum_{k=1}^{l} \sum_{s=1}^{i_{k}}\left\langle c_{k, s} \sin \left(\theta_{k, n, s}\right) w_{k, s}, v\right\rangle\right| \leq 2\|u\| \cdot\|v\| \cdot \sum_{k=1}^{l} \sum_{s=1}^{i_{k}}\left|\sin \left(\theta_{k, n, s}\right)\right|,
$$

which goes to zero as $n$ goes to infinity.

The subspace $Z_{l}^{\alpha_{n}}$ is nothing else but $\left(\begin{array}{c}I_{N} \\ V^{\left(\alpha_{n}\right)}\end{array}\right)$, while $W_{l+1}^{\alpha}$ is nothing else but $\left(\begin{array}{c}-V^{(\alpha)^{T}} \\ I_{M}\end{array}\right)$. The inner product of the $i$-th column vector of the first matrix and the $j$-th column vector of the second matrix is equal to

$$
V_{j i}^{\left(\alpha_{n}\right)}-V_{j i}^{(\alpha)}
$$

which must go to zero as $n$ goes to infinity. This proves the continuity of $V^{(\alpha)}$ on $\alpha$.

Lemma 11. Let $\Omega$ be the subset

$$
\begin{aligned}
& \left.\qquad\left(x_{j i}\right): \sum_{k=1}^{M} x_{k i}=-1, \forall 1 \leq i \leq N, \text { and } x_{j i} \leq 0, \forall 1 \leq j \leq M, 1 \leq i \leq N\right\} \\
& \text { of } \mathbb{R}^{M \times N} \text {. Then } V^{(\alpha)} \in \Omega \text { for all } \alpha \in[0,1] .
\end{aligned}
$$

Proof. Consider the subset

$$
\Gamma=\left\{\alpha \in[0,1): V^{(\alpha)} \in \Omega\right\}
$$

of the half-open half-closed interval $[0,1)$.

When $\alpha=0, v_{j i}^{(0)} \equiv-\frac{1}{M}$ (see Lemma 7 or equation (10). As a consequence, $V^{(0)} \in \Omega$, so that $0 \in \Gamma$ and $\Gamma$ is not empty.

Suppose there is a sequence of points $\alpha_{n} \in \Gamma$ and $\lim _{n \rightarrow \infty} \alpha_{n}=\alpha$ with $\alpha$ still in $[0,1)$. By Lemma 10, $\lim _{n \rightarrow \infty} V^{\left(\alpha_{n}\right)}=V^{(\alpha)}$. Because $\Omega$ is a compact set, so $V^{(\alpha)} \in \Omega$ and $\alpha \in \Gamma$. Therefore, $\Gamma$ is a closed subset of $[0,1)$.

Suppose $\alpha \in \Gamma$, namely $V^{(\alpha)} \in \Omega$ for some $\alpha \in[0,1)$. Because the quantities $\chi(i \sim j), f_{j}$ and $v_{j i^{\prime}}^{(\alpha)} v_{j^{\prime} i}^{(\alpha)}$ in equation (11) are all non-negative, we see that

$$
v_{j i}^{(\alpha)} \leq \frac{-(1-\alpha)}{(1-\alpha) M+\alpha\left(N+d_{i}\right)}<0 \text { for all } 1 \leq j \leq M, 1 \leq i \leq N .
$$

Therefore $V^{(\alpha)}$ is contained in the interior of $\Omega$. Since $V^{(\alpha)}$ depends continuously on $\alpha$, it follows that $\Gamma$ is an open subset of $[0,1)$.

The interval $[0,1)$ is connected, and $\Gamma$ is an open closed non-empty subset of it, therefore $\Gamma$ is equal to $[0,1)$.

By continuity at $\alpha=1, V^{(1)}$ is also in $\Omega$. This proves that $V^{(\alpha)} \in \Omega$ for all $\alpha \in[0,1]$. 
During the proof of Lemma 9 we have already known that

$$
L_{\alpha}\left(\begin{array}{c}
I_{N} \\
V^{(\alpha)}
\end{array}\right)=\left(\begin{array}{c}
I_{N} \\
V^{(\alpha)}
\end{array}\right) X_{\alpha}
$$

where

$$
X_{\alpha}=K_{N}+(1-\alpha) M+\alpha D_{1}-\left[(1-\alpha) J_{N \times M}+\alpha A\right] V^{(\alpha)} .
$$

So the sum of the $N$ largest eigenvalues of $L_{1}$ is equal to the trace of

$$
X_{1}=K_{N}+D_{1}-A V^{(1)} .
$$

But $V^{(1)} \in \Omega$ by Lemma 11, therefore

$$
\operatorname{Tr}\left(A V^{(1)}\right)=\sum_{i=1}^{N} \sum_{j: j \sim i} v_{j i} \geq \sum_{i=1}^{N} \sum_{j=1}^{M} v_{j i}=-N .
$$

Then

$$
\sum_{i=1}^{N} \lambda_{i}=N(N-1)+\operatorname{Tr}\left(D_{1}\right)-\operatorname{Tr}\left(A V^{(1)}\right) \leq N^{2}+\operatorname{Tr}\left(D_{1}\right) .
$$

By Proposition 5, this completes the proof of Lemma 6 .

\section{Proof of the Grone-Merris Conjecture}

For consistence we restate the Grone-Merris Conjecture here.

Grone-Merris Conjecture. For any graph $G$, its Laplacian spectrum is majorized by its conjugate degree sequence, namely $\lambda(G) \preccurlyeq \mathbf{d}^{\prime}(G)$.

The Grone-Merris Conjecture behaves nicely under complementation, in the sense of the proposition below.

The complement graph of a graph $G$ is a graph $\bar{G}$ on the same vertices such that two vertices of $\bar{G}$ are adjacent if and only if they are not adjacent in $G$. The Laplacian matrices of the graph $G$ and of its complementary graph $\bar{G}$ are related by the property that

$$
L(G)+L(\bar{G})+J_{n}=n I_{n} .
$$

All these matrices commute with each other, so that

$$
\begin{aligned}
\lambda(\bar{G}) & =\left(n-\lambda_{n-1}(G), \ldots, n-\lambda_{1}(G), 0\right) ; \\
\mathbf{d}^{\prime}(\bar{G}) & =\left(n-d_{n-1}^{\prime}(G), \ldots, n-d_{1}^{\prime}(G), 0\right) .
\end{aligned}
$$

From these we see that

Proposition 12. For any $1 \leq k<n$, the $k$-th inequality holds for the graph $G$ if and only if the $(n-k-1)$-th inequality holds for the complement graph $\bar{G}$,

$$
\sum_{i=1}^{k} \lambda_{i}(G) \leq \sum_{i=1}^{k} d_{i}^{\prime}(G) \Longleftrightarrow \sum_{j=1}^{n-1-k} \lambda_{j}(\bar{G}) \leq \sum_{j=1}^{n-1-k} d_{j}^{\prime}(\bar{G}), \quad \forall 1 \leq k<n .
$$

We are now ready to prove the Grone-Merris Conjecture.

Assume that the Grone-Merris Conjecture is not true, and the graph $G=(V, E)$ is a counterexample. Namely, there exists an integer $k$ with $1<k<n=|V|$, such that

$$
\sum_{i=1}^{k} \lambda_{i}>\sum_{i=1}^{k} d_{i}^{\prime}
$$


Without loss of generality, we can assume that this integer $k$ is minimum over all counterexamples. Then we have

$$
\sum_{i=1}^{k-1} \lambda_{i} \leq \sum_{i=1}^{k-1} d_{i}^{\prime}, \quad \text { and } \quad \lambda_{k}>d_{k}^{\prime} .
$$

Moreover, we can further assume that the number $|E|$ of edges is minimum over all counterexamples with the same $k$. Under this assumption, we claim that

Lemma 13. For any two vertices $i, j$ in the graph $G$, if $d_{i} \leq k$ and $d_{j} \leq k$, then they are not adjacent in $G$.

Proof. We will prove this by contradiction by assuming that the lemma is false. Namely there exists a pair of vertices such that

$$
d_{i} \leq k, \quad d_{j} \leq k, \quad i \sim j .
$$

Let $\widetilde{G}$ be the graph obtained from $G$ by deleting the edge $i j$. Due to the minimum property of $|E|$, we must have

$$
\sum_{i=1}^{k} \lambda_{i}(\widetilde{G}) \leq \sum_{i=1}^{k} d_{i}^{\prime}(\widetilde{G})
$$

Two Laplacian matrices are related via $L(G)=L(\widetilde{G})+H$, where $H_{n \times n}$ is a positive semi-definite matrix whose only non-zero entries are $H_{i i}=H_{j j}=1$ and $H_{i j}=H_{j i}=-1$. Applying Fan's Theorem 3 , we see that

$$
\begin{aligned}
\sum_{i=1}^{k} \lambda_{i}(G) & \leq \sum_{i=1}^{k} \lambda_{i}(\widetilde{G})+\sum_{i=1}^{k} \lambda_{i}(H) \leq \sum_{i=1}^{k} d_{i}^{\prime}(\widetilde{G})+\operatorname{Tr}(H) \\
& =\left[\sum_{i=1}^{k} d_{i}^{\prime}(G)-2\right]+2=\sum_{i=1}^{k} d_{i}^{\prime}(G) .
\end{aligned}
$$

This contradicts our assumption that $G$ was a counterexample, and therefore concludes the proof.

Next, we add new edges to $G$ to get a new graph $\widehat{G}$. Add to $G$ a new edge $i j$ for any pair of vertices $i, j$ in $G$ such that

$$
d_{i} \geq k, d_{j} \geq k, \text { and } i \nsim j .
$$

The new graph $\widehat{G}$ so obtained is a split graph.

The clique of $\widehat{G}$ consists of all vertices of $G$ whose degree is at least $k$, so the size of the clique is equal to $d_{k}^{\prime}(G)$. Let $N=d_{k}^{\prime}(G)$ denote this size. The co-clique consists of all vertices of $G$ whose degree is less than $k$, so the maximum degree of vertices in the co-clique is $\delta(\widehat{G}) \leq k-1$.

Note that

$$
d_{1}^{\prime}(\widehat{G})=d_{1}^{\prime}(G), \ldots, d_{k}^{\prime}(\widehat{G})=d_{k}^{\prime}(G)
$$

while $\lambda_{i}(\widehat{G}) \geq \lambda_{i}(G)$ for all $1 \leq i \leq n$, so these two inequalities are still valid for the new graph $\widehat{G}$, namely

$$
\sum_{i=1}^{k} \lambda_{i}(\widehat{G})>\sum_{i=1}^{k} d_{i}^{\prime}(\widehat{G}) \quad \text { and } \quad \lambda_{k}(\widehat{G})>d_{k}^{\prime}(\widehat{G})=N .
$$

Let us discuss the relationship between $N$ and $k$. 
If $N<k$, then $\lambda_{k}(\widehat{G}) \leq \lambda_{N+1}(\widehat{G}) \leq N$, which leads to a contradiction. The second inequality comes from Proposition 5

If $N=k$, then $\widehat{G}$ is a split graph of clique size $N$, with the property that

$$
\sum_{i=1}^{N} \lambda_{i}(\widehat{G})>\sum_{i=1}^{N} d_{i}^{\prime}(\widehat{G}) \quad \text { and } \quad \lambda_{N}(\widehat{G})>N .
$$

This contradicts Lemma 6 .

So $k<N$. Note that $\widehat{G}$ is a split graph of clique size $N$. In this graph $\widehat{G}$, the maximum degree of vertices in the co-clique is at most $(k-1)$, while the minimum degree of vertices in the clique is at least $(N-1)$. This means that

$$
d_{N-1}^{\prime}(\widehat{G})=\cdots=d_{k+1}^{\prime}(\widehat{G})=d_{k}^{\prime}(\widehat{G})=N .
$$

Combining this with $\lambda_{k+1}(\widehat{G}) \geq \ldots \geq \lambda_{N-1}(\widehat{G}) \geq N$ from Proposition 5 , we see immediately that the inequality

$$
\sum_{i=1}^{k} \lambda_{i}(\widehat{G})>\sum_{i=1}^{k} d_{i}^{\prime}(\widehat{G}) \text { can be extended to } \sum_{i=1}^{N-1} \lambda_{i}(\widehat{G})>\sum_{i=1}^{N-1} d_{i}^{\prime}(\widehat{G}) .
$$

Then we proceed to compare $\lambda_{N}(\widehat{G})$ with the clique size $N$.

First consider the case where $\lambda_{N}(\widehat{G}) \geq N$. Because $N=d_{N-1}^{\prime}(\widehat{G}) \geq d_{N}^{\prime}(\widehat{G})$, the split graph $\widehat{G}$ has clique size $N$, with the additional property that

$$
\sum_{i=1}^{N} \lambda_{i}(\widehat{G})>\sum_{i=1}^{N} d_{i}^{\prime}(\widehat{G}) \quad \text { and } \quad \lambda_{N}(\widehat{G}) \geq N>\delta(\widehat{G}) .
$$

This again contradicts Lemma 6

In the other case, where $\lambda_{N}(\widehat{G})<N$, we switch attention to the complement graph of $\widehat{G}$. This complement graph is another split graph $\overline{\widehat{G}}$. Its clique size is $M$, and

$$
\lambda_{M}(\overline{\widehat{G}})=(N+M)-\lambda_{N}(\widehat{G})>M .
$$

According to Proposition 12 ,

$$
\sum_{i=1}^{N-1} \lambda_{i}(\widehat{G})>\sum_{i=1}^{N-1} d_{i}^{\prime}(\widehat{G}) \Longrightarrow \sum_{i=1}^{M} \lambda_{i}(\widehat{\widehat{G}})>\sum_{i=1}^{M} d_{i}^{\prime}(\widehat{\widehat{G}}) .
$$

Therefore, $\overline{\widehat{G}}$ is a split graph of clique size $M$, with the additional property that

$$
\sum_{i=1}^{M} \lambda_{i}(\overline{\widehat{G}})>\sum_{i=1}^{M} d_{i}^{\prime}(\overline{\widehat{G}}) \quad \text { and } \quad \lambda_{M}(\overline{\widehat{G}})>M .
$$

This again contradicts Lemma 6

All possible cases are eliminated, and the Grone-Merris Conjecture is proved.

\section{ACKNOWLEDGEMENTS}

This work was started while the author was visiting the University of Southern California, whose support and hospitality is gratefully acknowledged. The author also thanks Francis Bonahon for his support and encouragement throughout the years, Jun Ying and Jie Ying for critical Matlab computations, Russell Merris for useful suggestions, and Andries Brouwer, Tao Li and the referee for many valuable comments. 


\section{REFERENCES}

[1] Ravindra B. Bapat, Arbind K. Lal and Sukanta Pati, Laplacian spectrum of weakly quasithreshold graphs, Graphs Comb. 24, no. 4 (2008), 273-290. MR2438859 (2009g:05099)

[2] Fan R. K. Chung, Spectral graph theory, volume 92 of CBMS Regional Conference Series in Mathematics, American Mathematical Society, 1997. MR:1421568 (97k:58183)

[3] Art M. Duval and Victor Reiner, Shifted simplicial complexes are Laplacian integral, Trans. Amer. Math. Soc. 354 (2002), 4313-4344. MR.1926878 (2003j:15017)

[4] Ky Fan, On a theorem of Weyl concerning eigenvalues of linear transformations I, Proc. Nat. Acad. Sci. USA 35 (1949), 652-655. MR0034519(11:600e)

[5] Stéhane Földes and Peter L. Hammer, 'Split graphs', Proceedings of the Eighth Southeastern Conference on Combinatorics, Graph Theory and Computing, 1977, Congressus Numerantium, XIX, Winnipeg: Utilitas Math., 311-315. MR0505860 (58:21844)

[6] David Gale, A theorem on flows in networks, Pacific J. Math. 7 (1957), 1073-1082. MR0091855 (19:1024a)

[7] Robert Grone, Eigenvalues and degree sequences of graphs, Lin. Multilin. Alg. 39 (1995) 133-136. MR1374475 (97a:05148)

[8] Robert Grone and Russell Merris, Coalescence, majorization, edge valuations and the Laplacian spectra of graphs, Lin. Multilin. Alg. 27, No.2 (1990), 139-146. MR1054137|(91c:05129)

[9] Robert Grone and Russell Merris, The Laplacian spectrum of a graph II, SIAM J. Disc. Math. 7 (1994), 221-229. MR1271994 (95d:05085)

[10] Peter L. Hammer and Bruno Simeone, The spittance of a graph, Combinatorica 1 (1981), 275-284. MR637832(84c:05050)

[11] Roger Alfred Horn, Doubly stochastic matrices and the diagonal of a rotation matrix, Amer. J. Math. 76 (1954), 620-630. MR.0063336 (16:105c)

[12] Nets Hawk Katz, The Grone Merris conjecture and a quadratic eigenvalue problem, preprint, 2005; arXiv:math.CA/0512647.

[13] Steve Kirkland, Near threshold graphs, Electron. J. Combin. 16, no. 1(2009), Research Paper R42. MR2491644 (2010g:05221)

[14] Russell Merris, Laplacian matrices of graphs: a survey, Linear Algebra and its Applications $197 \& 198$ (1994), 143-176. MR.1275613 (95e:05084)

[15] Russell Merris, Split graphs, European J. Combin. 24, 4 (2003), 413-430. MR1975945 (2004b:05064)

[16] Michael Reed and Barry Simon, Methods of Modern Mathematical Physics IV: Analysis of Operators, Academic Press, 1978. MR0493421 (58:12429c)

[17] Herbert J. Ryser, Combinatorial properties of matrices of zeros and ones, Pacific J. Math. 7 (1957), 1073-1082. MR0087622 (19:379d)

[18] Issac Schur, Uber eine Klasse von Mittelbidungen mit Anwendungen die Determinanten, Sitzungsber. Berlin. Math. Gesellschaft 22 (1923), 9-20.

[19] Tamon Stephen, On the Grone-Merris conjecture, DMTCS proc. AE (2005), 187-192.

[20] Regina I. Tyshkevich and Arkady A. Chernyak, Canonical partition of a graph defined by the degrees of its vertices, (in Russian), Isv. Akad. Nauk BSSR, Ser. Fiz.-Mat. Nauk 5 1979, 14-26. MR.554162 (81f:05098)

Department of Mathematics, Boston College, Chestnut Hill, Massachusetts 02467

E-mail address: baihu@bc.edu

E-mail address: huabai@alumni.usc.edu 